\title{
COMPARISON OF SHEAR BOND STRENGTH OF TWO BONDING AGENTS FOR RECYCLED SELF-LIGATING BRACKETS
}

\author{
Ghada El-Mehy*
}

\begin{abstract}
Introduction: Bracket dislodgment is a common orthodontic problem. The clinician might has the option of reusing the old bracket after recycling rather than replacing it with a new one.

Purpose: This current invitro study was designed to compare the effect of recycling of selfligating brackets on shear bond strength (SBS) of both flowable composite and self-etch primer.

Materials \& Methods: Thirty extracted premolars were randomly divided into two groups of 15 teeth per each according to the type of adhesive used. In group I, self-ligating (Damon) Brackets were bounded with flowable composite Grandio Flow (GF) and in group II were bonded with Transbond plus self-etch primer (SEP). All the specimen were subjected to recycling by a low speed carbide debonding burs. The Universal Testing Machine was used to measure SBS. The mode of bond failure was evaluated by the adhesive remnant index (ARI). The data was analysed with T-test while ARI scores were evaluated by Wilcoxon test.
\end{abstract}

Results: There was a significant difference between the two tested groups $(\mathrm{P}<0.05)$. Flowable composite (GF) showed a significantly higher SBS than self-etching primer (SEP), for both before and after recycling. After recycling there was a nonsignificant difference in SBS of the two groups $(P>0.05)$, for ARI, there was a nonsignificant difference between the two tested groups $(P>0.05)$.

Conclusion: Self-ligating Damon brackets bonded with (GF) and (SEP) could be successfully recycled by low speed debonding bur. GF was superior in SBS.

KEY WORDS: bond strength, recycling, self ligating brackets, adhesives.

\section{INTRODUCTION}

Bond failure of orthodontic brackets during the course of treatment is a common occurrence, whatever the technique or the adhesive used ${ }^{(1-2)}$. The majority of bond failure is the result of technique sensitivity. It might be due to poor moisture control. (3) Incomplete etching due to deficient cleaning of the tooth surface before etching, ${ }^{(4)}$ variation in etching time and inadequate curing time were also found to affect SBS..$^{(5)}$ Moreover, it may

*ASS. Prof. of Orthodontics, Faculty of Dentistry, Tanta University. 
occurs if the patients did not follow the restricted diet as instructed by the clinician. ${ }^{(4)}$ Another cause of bond failures related to the initial tooth position. ${ }^{(6)}$

The mode of bond failure may be in a combination of adhesive and/or cohesive failure. Adhesive failures occur between two materials, while cohesive failures occur within one material. It may be at the enamel/ adhesive interface, at the bracket-adhesive interface and within the adhesive resin. ${ }^{(7)}$

The clinicians have to take the decision about what to do with the dislodged or inaccurately positioned brackets. Weather to use a new one or recycle the old bracket. ${ }^{(8-9)}$ Recycling seems to be an alternative to facilitate the reuse of the dislodged brackets. ${ }^{(10)}$

The clinically accepted SBS is ranging between 6 and $8 \mathrm{MPa}$ to obtain are liable and efficient adhesive bond between orthodontic brackets and enamel surface ${ }^{(11)}$. Recycling of the brackets should achieve SBS comparable to that of the clinically accepted values. SBS of the recycled brackets is affected by several factors including the type of bracket and adhesive used, the amount of adhesive remnant, the method used for adhesive removal and the design of the brackets base. ${ }^{(12)}$

Various instruments and procedures were introduced to facilitate rebonding with an efficient, simple and safe method of adhesive removal. These includes mechanical methods as tungsten carbide burs, ${ }^{(13-14)}$ diamond burs, ${ }^{(15)}$ sandplasting ${ }^{(13)}$ and soflex disc ${ }^{(16)}$. There are also thermal, chemical methods $^{(17)}$ and laser recycling ${ }^{(18)}$.

From the economic point of view, it is valuable to recycle the relatively expensive Damon brackets, without interfering with the quality. Therefore this study was conducted to compare SBS of GF and SEP for recycled Demon brackets, which might improve and simplify the bonding procedure.

\section{AIM OF THE WORK}

The aim of the present study was to compare the SBS of (GF) and (SEP) used for recycled self-ligating (Damon) brackets. Also to evaluate the failure mode.

\section{MATERIALS AND METHODS:}

This current invitro study was carried out on thirty extracted first premolars for orthodontic purposes. The post-hoc power percentage $(97.60 \%)$ indicated that the power of sample size was adequate (tabel.1)

TABLE (1): Post-hoc power analysis of sample size for SBS analysis.

\begin{tabular}{|c|c|c|c|}
\hline Group & Number & Mean \pm S.D & Post-hoc Power \\
\hline Group I & 15 & $2.42 \pm 18.72$ & \multirow{2}{*}{$\% 97.60$} \\
\hline Group II & 15 & $2.05 \pm 15.61$ & \\
\hline
\end{tabular}

This study was done according to the Orthodontic Department research plane which approved by the Faculty of Dentistry, Tanta University's council.

The selection criteria included, intact buccal enamel surface without any defect or restoration and caries free, the collected teeth were rinsed and kept in saline solution at room temperature, which was changed every two weeks. The buccal enamel surface of each tooth was bonded with Damon 3MX self - ligating brackets (Ormco corporation, Orange Co., CA, USA).The enamel surface of each tooth was polished with fluoride-free pumice and rubber cup for 10 seconds, sprayed with water and dried with compressed oil-free stream.

The samples were randomly divided into two equal groups according to the adhesive system used into group (1) and group (2). All steps were performed according to the manufacturer's instructions. 
In group (1) ( $\mathrm{n}=15)$ was etched with $37 \%$ phosphoric acid for 30 seconds, rinsed and dried then sealed with flowable composite resin, Grando Flow (GF) (Voco, Cuxhaven, Germany) with TXT primer, GF was applied following TXT protocol.

In group (2) (n = 15) was sealed with transbond Plus Self - Etching primer (3 Munitek, South Peck Road, Morovia, USA) which etch and prime the teeth at the same time without separate etching agent.

Each bracket was positioned on the buccal surface, in the middle third of the crown, it was pressed firmly and excess resin was removed using a sharp scalar and light cured for 20 seconds.

All the specimens were mounted in an acrylic blocks with the crown only exposed, the acrylic blocks were color coded according to the study groups, group (1) taking pink colour and group (2) taking green colour. SBS was measured for both the two tested groups before and after recycling process by a Universal Testing Machine at the Department of Dental Biomaterials, Faculty of Dentistry, Tanta University (fig.1). Each sample was fixed to stable lower part of the machine. A sharp steel blade was secured to the mobile upper part of the device. Each sample was subjected to a compressive shear load at across head speed of $1 \mathrm{~mm} . / \mathrm{min}$. till failure. The force needed to displace every bracket was calculated in Newton and the SBS was estimated by dividing the load by the surface area of the bracket base to obtain SBS in megapascal (MPa).

After initial debonding and SBS measurements all specimen were recycled by removing the adhesive from bracket base and enamel surface using low speed carbide debonding bur ( $\mathrm{db}$ orthodontics. co.uk) (fig.2). A new bure was used every 5 samples. The adhesive remnant on the enamel surface was removed until it becomes glossy without any visible composite, rebonding was again carried out of enamel with the same previous method and then debonding was done with the Universal Testing
Machine for measuring the SBS of the recycled brackets.

The quantity of the residual adhesive on each tooth was assessed according to adhesive remnant index (ARI) as described by Arturn \& Bergland ${ }^{(19)}$. The bracket base and enamel surface were examined under 10x magnification and given score from 0 to 3 as follow:

- Score 0: no adhesive left on the tooth surface.

- Score 1: less than half of the adhesive left on the tooth surface.

- Score 2: more than half of the adhesive left on the tooth surface.

- Score 3: all the adhesive left on the tooth surface with distinct impression of the bracket base.

\section{Statistical analysis:}

The collected data was statistically analyzed. Mean and standard deviation of SBS were assessed using SPSS, statistical package for social sciences, version 24. T-test was utilized to compare SBS between the tested groups before and after recycling and Wilcoxon test was assessed for evaluation of ARI. Differences were considered significant at $\mathrm{P}<0.05$.

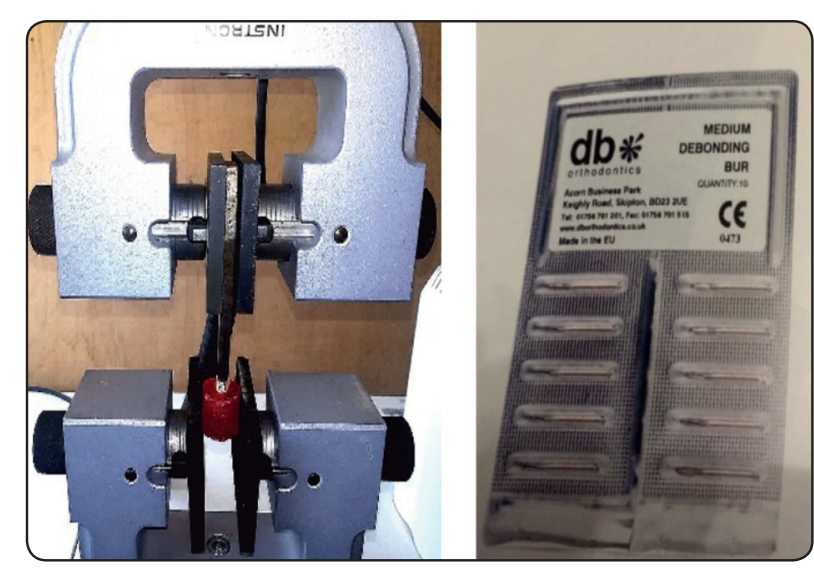

Fig. (1) Universal Testing Machine. Fig. (2) Debonding burs 


\section{RESULTS}

Descriptive statistics of SBS of both groups was demonstrated in table (2). Paired t-test showed that there was a non significant difference $(\mathrm{P}>0.05)$ of SBS before and after recycling in the two tested groups (table 2). Independent t-test showed that there was a significant difference of SBS $(\mathrm{P}<0.05)$ between the two tested groups before and after recycling (table 3 ), with the higher mean value of
SBS of group 1 (before recycling 18.72MPa \& after recycling $17.40 \mathrm{MPa}$ ) than that of group 2 (before recycling 15.61 MPa\& after recycling 14.13 $\mathrm{MPa}$ ).

Concerning ARI scores, statistical analysis of Wilcoxon- test showed that there was a non significant difference $(\mathrm{P}>0.05)$ among all the tested groups before and after recycling, with the higher frequency of score 1 and 2 (table 4 ).

TABLE (2) Descriptive statistics and paired t-test of SBS before and after recycling.

\begin{tabular}{|c|c|c|c|c|c|c|}
\hline \multicolumn{2}{|c|}{ S.B.S } & Mean \pm S.D & Median & Range & t-value & p-value \\
\hline \multirow{2}{*}{ Group I } & Before & $2.42 \pm 18.72$ & 19.40 & $23.20-15.20$ & \multirow{2}{*}{1.829} & 0.079 \\
\cline { 2 - 5 } & After & $2.10 \pm 17.40$ & 17.30 & $21.30-12.60$ & & \multirow{2}{*}{2.027} \\
\hline \multirow{2}{*}{ Group II } & Before & $2.05 \pm 15.61$ & 16.50 & $19.70-12.56$ & 0.062 \\
\cline { 2 - 7 } & After & $1.87 \pm 14.13$ & 14.60 & $17.30-10.20$ & & \\
\hline
\end{tabular}

TABLE (3) Independent t-test between group I and group II of SBS between the two tested groups before and after recycling.

\begin{tabular}{|c|c|c|c|c|}
\hline \multirow{2}{*}{ Before } & Group I & Mean \pm S.D & t-value & p-value \\
\cline { 2 - 3 } & Group II & $2.42 \pm 18.72$ & \multirow{2}{*}{3.798} & $* 001$ \\
\hline \multirow{2}{*}{ After } & Group I & $2.05 \pm 15.61$ & \multirow{2}{*}{$* 0.000$} \\
\cline { 2 - 3 } & Group II & $1.87 \pm 14.13$ & 4.504 & $* 0.00$ \\
\hline
\end{tabular}

*Significance at $P<0.05$

TABLE (4) Wilcoxon test of (ARI) if the two studied groups before and after recycling.

\begin{tabular}{|c|c|c|c|c|c|c|c|}
\hline \multirow{2}{*}{\multicolumn{2}{|c|}{$\begin{array}{c}\text { Groups } \\
(\%) \mathrm{N}\end{array}$}} & $0=\mathrm{ARI}$ & $1=\mathrm{ARI}$ & $2=\mathrm{ARI}$ & $3=\mathrm{ARI}$ & \multirow{2}{*}{$\mathrm{Z}$} & \multirow{2}{*}{ p-value } \\
\hline & & $(\%) \mathrm{N}$ & $(\%) \mathrm{N}$ & $(\%) \mathrm{N}$ & & & \\
\hline \multirow{2}{*}{ Group I } & Before & $(\% 20) 3$ & $(\% 53.3) 8$ & $(\% 26.7) 4$ & $(\% 0) 0$ & \multirow{2}{*}{1.249} & \multirow{2}{*}{0.905} \\
\hline & After & $(\% 13.3) 2$ & $(\% 40) 6$ & $(\% 40) 6$ & $(\% 6.7) 1$ & & \\
\hline \multirow{2}{*}{ Group II } & Before & $(\% 20) 3$ & $(\% 46.7) 7$ & $(\% 33.3) 5$ & $(\% 0) 0$ & \multirow{2}{*}{0.212} & \multirow{2}{*}{0.366} \\
\hline & After & $(\% 6.7) 1$ & $(\% 53.3) 8$ & $(\% 33.3) 5$ & $(\% 6.7) 1$ & & \\
\hline
\end{tabular}




\section{DISCUSSION}

There is a trend in orthodontics to simplify the technical procedures, reduce operative time and treatment coast, but without interference with the efficiency. Hence, this study was performed to compare SBS of the recycled Damon brackets using GF composite and SEP. In this study all the brackets were bonded then debonded and SBS was recorded as a control for the recycling process as performed by Andrew et al (20).

The use of Damon self- ligating brackets with low friction and light force helps in improving treatment quality, increasing patient's comfort, decreasing treatment time and minimal chair-time ${ }^{(21)}$, also with accepted $\mathrm{SBS}^{(22)}$. So, the relatively expensive Damon brackets, were recycled in this study.

Among the several introduced orthodontic adhesives, SEP was found to simplify the bonding procedures, as it combined both the conditioner and the primer into a single solution thus reducing the clinical steps. ${ }^{(23)}$ Also, GF produces desirable clinical advantage because of its fluid injectability, high flexibility and low modulus of elasticity. ${ }^{(24)}$

There are different techniques for adhesive removal from the bracket base and enamel surface but without detection which produces optimal bond strength and minimal damage to the enamel surface $^{(3)}$. The use of in-office method for time consuming as low speed debonding carbide bur was considered as a simple, fast, efficient and economic method of adhesive removal.

The results of this laboratory study revealed that self-ligating Damon brackets could be successfully recycled by debonding bur at low speed with a nonsignificant difference in SBS between the two groups of bonding agents with the higher SBS of GF composite over SEP before and after recycling. This is in accordance with Behnam et al ${ }^{(25)}$. who reported that using tungsten carbide bur at high and low speed was comparatively superior with nonsig- nificant difference in SBS of the recycled brackets, while sandplasting was time consuming and damaging the enamel surface even it might produce sufficient SBS. Also Bayram et al ${ }^{(15)}$ reported that bracket recycling with diamond bur was effective and resulted in accepted SBS. This might be due to the less abrasion effect of the retentive mesh of the bracket when using low speed debonding bur. On the other hand, other studies considered recycling with adhesive grinding methods produce significant reduction in SBS. ${ }^{(26-27)}$

In agreement with this study, GF showed a higher SBS ${ }^{(28)}$.The difference in SBS of the studied adhesives might be due to the difference in composition and bonding affinity. GF with TXT primer contains inorganic filler, thus producing small contact angels, good wetting, preventing formation of air voids and resulting in high penetration coefficient with both the enamel surface and brackets mesh . It was recommended that SEP showed a lower but clinically accepted SBS ${ }^{(29)}$, this might be attributed to the lesser retention micromechanical undercuts produced by SEP adhesive system.

In this study the failure mode or ARI showed a nonsignificant difference among the tested groups before and after recycling with a higher frequency of score 1 and 2, this indicate a mixed adhesion modality. This is on line with the work of Sfondrini et al ${ }^{(22)}$

It has to be remembered that all the invitro laboratory studies of SBS could not predict clinical performance but could be considered only as a screening tool ${ }^{(30) \text {. }}$

\section{CONCLUSION}

Recycling of self- ligating Damon brackets using low speed debonding bur was efficient, simple, time saving and provide adequate SBS, with cost benefit for both orthodontist and patient, using flowable composite (GF) might be comparatively superior over self-etching primer (SEP) in SBS. 


\section{RECOMMENDATIONS}

Further studies are required to evaluate the longterm clinical performance of the recycled Self- Ligating Demon brackets and the changes of enamel surface after recycling.

\section{REFERENCES}

1. Sonis AL. Air abrasion of failed bonded metal brackets: A study of shear bond strength and surface characteristics as determined by scanning electron microscopy. Am J Orthod Dent of a cOrthop. 1996; 110:96-98.

2. Sukhia R H, Sukhia H R, Mahar A.Bracket debonding \& breakage prevelance in orthodontic patients. Pakistan Oral Dent J. 2011; 31: 73-77.

3. Hormati AA, Fuller JL, Denehy GE. Effects of contamination and mechanical disturbance on the quality of acidetched enamel. J Am Dent Assoc. 1980; 100:34-38.

4. O'Brien KD, Read MJ, Sandison RJ, Roberts CT. A visible light-activated direct-bonding material: An in vivo comparative study. Am J Orthod Dentofac Orthop. 1989;95:348-51.

5. Johnston C D, Hussey DL, Burden DJ. The effect of etch duration on the micromorphology of molar enamel: an in vitro study. Am J Orthod DentofacOrthop. 1996;109: 531-34

6. Živko-Babić J, Pandurić J, Jerolimov V, Mioč M, Pižeta I, Jakovac M. Bite Force in subjects with complete Dentition. Coll. Antropol. 2002; 1: 293-302

7. Vicente A,Toledano M, Bravo AL, Romeo A, Higuera B, Osorio R. Effect of water contamination on the shear bond strength of five orthodontic adhesives. Med Oral Patol Oral Cir Bucal. 2010;15: 820-26.

8. Cacciafesta V, Sfondrini MF, Melsen B, Dcribante A. A 12 month clinical study of bond failures of recycled versus new stainless steel orthodontic brackets. Eur J Orthod.2004; 26: 449-54.

9. Wright WL, Powers JM. In vitro tensile bond strength of reconditioned brackets. Am J Orthod Dentofac Orthop. $1985 ; 87: 247-52$.

10. Grabouski JK, Staley RN, Jakobsen JR. The effect of micro etching on the bond strength metal brackets when bonded to previously bond teeth: an in vitro study. Am J Orthod Dentofac Orthop.1998; 113: 452-60.
11. Reynolds IR. A review of direct orthodontic bonding. Br J Orthod 1975; 2: 171-8.

12. Sharma-Sayal SK, Rossouw PE, Kulkarni GV, TitleyKC. The influence of orthodontic bracket base design on shear bond strength. Am J Orthod Dentofac Orthop.2003; 124:74-82.

13. Eminkahyagil N., Arman A., Getinsahin A, Karabulat E. Effect of resin- removal methods on enamel and shear bond strength of rebonded beackets. Angle Orthod,2006; 76:314-21.

14. Eliades T, Gioka C, Eliades G, Makou M, Enamel Surface roughiness following debonding during two resine grinding methods. Eur. J orthod. 2004; 26: 333-8.

15. Bayram M, Yesilyurt C, Kusgoz A, Ulker M, Nur M. Shear bond strength of orthodontic brackets to aged resin composite surfaces: Effect of Surface conditioning. Eur J orthod. $2011 ; 33$ : 174-9.

16. Howell S, Weekes WT. An electronmicroscopic evaluation of the enamel Surface subsequent to varios debonding procedures. Aust Dent J 1990; 35: 245-52.

17. Basudan AM, Al-Emran SE. The effect of in-office reconditioning on the morphology of slots and bases of stainless steel brackets and on thes hear/peel bond strength. J Orthod. 2001;28:231-36.

18. Yassaei S, Aghili H, KhanPayeh E, Goldani Moghadam M. Comparison of shear bond strength of rebonded brackets with four methods of adhesive removal. Lasers Med Sci.2014; 29:1563-68.

19. Artun J, Bergland S: Clinical trails with crystal growth conditioning as an alternative to acid-etch enamel pretreatment. Am J Orthod. 1984; 85:333-340.

20. Andrew N Quick, Angela MP Harris, Vince P Joseph. Office reconditioning of stainless steel orthodontic attachment. The Eur. J of Orthod 2005, 27 (3): 231-236.

21. Harradine NW. Self- ligating brackets: where are now? Journal of orthod-2003, 30: 262-273.

22. Maria Francesca Sfondrini, Gatti S., Scribante A: shear bond strength of self-ligating brackets. European Journal of Orthodontics, 2011, 33, 1: 71-74.

23. Yamada R, Hayakawa T, Kasai K: effect of using selfetching primer for bonding orthodontic brackets. Angle Orthod. 2002; 72 (6): 558-564. 
24. Ferracane J. Developing a more complete understanding of stresses produced in dental composites during polymerization. J Dent Mater. 2005; 21:36-42.

25. Behnam Kh., Soed N.A, Shar N. and Vahid R. Assessing the effect of three resin removal methods and bracket sand plasting on shear bond strength of metallic orthodontic brackets and enamel surface. Orthodontic waves, 2011, 70,1:27-38.

26. Ahrari F, Poosti M, abkari M, Sadrik. Early versus delayed rebonding of orthodontic bracket. Prog. Orthod. 2012; 13: 17-22.

27. Demirates HK, Akin M, Ileri Z, Basciftci FA. Shear bond strength of orthodontic brackets to aged nanohybrid com- posite resin surface using different surface preparation. Dent. Mater J 2015; 34: 86-90.

28. Park S., Son W., Koc, Garci F, Park M, Kim H. and Kwonyy. Influence of flowable resins on shear bond strength of orthodontic bracktes resins. Dent Mater. J. 2009; 28 (6): 730-734.

29. Bishara S.E, Ajlouni R., Laffoon JF, Waren JJ. Effect of a fluoride- releasing self-etch acidic primer on shear bond strength of orthodontic brackets. Angle orthod. 2002; 72; 199-202.

30. Eliades T., Lekka M., Eliades G., Brantly W.A.: surface characterization of ceramic brackets: a multi-technique approach. Am.J.Orthod. Dentofac. Orthop 1994; 105: 10-18. 